\title{
Defunct brain stem cardiovascular regulation underlies cardiovascular collapse associated with methamphetamine intoxication
}

\author{
Faith CH Li ${ }^{1,2}, J C$ Yen$^{2}$, Samuel HH Chan ${ }^{1}$ and Alice YW Chang ${ }^{1 *}$
}

\begin{abstract}
Background: Intoxication from the psychostimulant methamphetamine (METH) because of cardiovascular collapse is a common cause of death within the abuse population. For obvious reasons, the heart has been taken as the primary target for this METH-induced toxicity. The demonstration that failure of brain stem cardiovascular regulation, rather than the heart, holds the key to cardiovascular collapse induced by the pesticide mevinphos implicates another potential underlying mechanism. The present study evaluated the hypothesis that METH effects acute cardiovascular depression by dampening the functional integrity of baroreflex via an action on brain stem nuclei that are associated with this homeostatic mechanism.
\end{abstract}

Methods: The distribution of METH in brain and heart on intravenous administration in male Sprague-Dawley rats, and the resultant changes in arterial pressure (AP), heart rate (HR) and indices for baroreflex-mediated sympathetic vasomotor tone and cardiac responses were evaluated, alongside survival rate and time.

Results: Intravenous administration of METH (12 or $24 \mathrm{mg} / \mathrm{kg}$ ) resulted in a time-dependent and dose-dependent distribution of the psychostimulant in brain and heart. The distribution of METH to neural substrates associated with brain stem cardiovascular regulation was significantly larger than brain targets for its neurological and psychological effects; the concentration of METH in cardiac tissues was the lowest among all tissues studied. In animals that succumbed to METH, the baroreflex-mediated sympathetic vasomotor tone and cardiac response were defunct, concomitant with cessation of AP and HR. On the other hand, although depressed, those two indices in animals that survived were maintained, alongside sustainable AP and HR. Linear regression analysis further revealed that the degree of dampening of brain stem cardiovascular regulation was positively and significantly correlated with the concentration of METH in key neural substrate involved in this homeostatic mechanism.

Conclusions: We conclude that on intravenous administration, METH exhibits a preferential distribution to brain stem nuclei that are associated with cardiovascular regulation. We further found that the concentration of METH in those brain stem sites dictates the extent that baroreflex-mediated sympathetic vasomotor tone and cardiac responses are compromised, which in turn determines survival or fatality because of cardiovascular collapse.

\section{Background}

The psychostimulant methamphetamine (METH; Nmethyl-O-phenylisopropylamine) is among the most popular illegal drugs worldwide [1-4]. Based on records between 1998 and 2001 from the Ministry of Justice, Taiwan, it has been reported that this schedule II

\footnotetext{
* Correspondence: cgmf.kmc@gmail.com

${ }^{1}$ Center for Translational Research in Biomedical Sciences, Kaohsiung Chang

Gung Memorial Hospital, Kaohsiung 833, Taiwan, Republic of China

Full list of author information is available at the end of the article
}

controlled drug is being used by approximately $79.3 \%$ of drug offenders in Taiwan [5]. Because METH enhances alertness, concentration, energy, euphoria and libido, it has become a well-known drug of abuse that leads to severe societal and economical consequences $[6,7]$. The best known pharmacological effects of METH are well documented to be associated with brain sites that are involved in the reward system [8-11] and neurodegenerative disease [11-13]. As such, nucleus accumbens (NACC), globus pallidas (GPi), caudate nucleus (Cd),

\section{Biomed Central}


substantia nigra ( $\mathrm{SN}$ ) and ventral tegmental nucleus (VTA) are among the most often mentioned brain targets for METH-induced psychological or neurological responses [8-13].

METH intoxication is a common cause of death within the abuse population $[14,15]$. In particular, severe hypotension and bradycardia are often observed in patients who exhibit acute METH intoxication $[1,15,16]$, with $100 \%$ mortality despite intensive care in a hospital setting $[1,17]$. For obvious reasons, the heart has been taken as the primary target for METH-induced cardiovascular toxicity. Thus, atrioventricular arrhythmia and myocardial ischemia reportedly contribute to METHinduced fatality [18]. Of note is that our laboratory [19] demonstrated recently that failure of brain stem cardiovascular regulation, rather than the heart, holds the key to cardiovascular collapse induced by the pesticide mevinphos. This suggests that another target for METH-induced cardiovascular toxicity may be brain stem nuclei, including nucleus tractus solitarii (NTS) and rostral or caudal ventrolateral medulla (RVLM or CVLM) that are associated with the baroreflex, which is responsible for the maintenance of stable blood pressure and heart rate [20].

The present study evaluated the hypothesis that METH effects acute cardiovascular depression by dampening the functional integrity of baroreflex via an action on brain stem nuclei that are associated with this homeostatic mechanism. Based on evaluations of distribution of METH in brain and heart on intravenous administration, baroreflex responses and survival rate and time, this hypothesis was validated.

\section{Methods}

Adult male Sprague-Dawley rats $(275-315 \mathrm{~g}, \mathrm{n}=39)$ purchased from the Experimental Animal Center of the National Science Council and BioLASCO, Taiwan, Republic of China were used. Rats were housed in an Association for Assessment and Accreditation of Laboratory Animal Care (AAALAC) International-accredited animal facility under temperature control (24-25 C) and 12-h light-dark cycle. Standard laboratory rat chow and tap water were available ad libitum. All experimental procedures carried out in this study were approved by the Institutional Animal Care and Use Committee of the Kaohsiung Chang Gung Memorial Hospital (CGMH891071). Efforts were made to reduce the number of animals used and to minimize animal suffering during the experiment.

\section{General preparation}

Under an induction dose of pentobarbital sodium (50 $\mathrm{mg} / \mathrm{kg}$, i.p.), animals received preparatory surgery that included tracheal intubation and cannulation of the femoral artery and vein. During the recording session, which routinely commenced $60 \mathrm{~min}$ after the administration of pentobarbital sodium, animals received an intravenous infusion of propofol $(20-25 \mathrm{mg} / \mathrm{kg} / \mathrm{h}$; Zeneca, Macclesfield, England), which provided satisfactory maintenance of anesthesia while preserving the capacity of central cardiovascular regulation [21]. Animals were allowed to breathe spontaneously with room air, and body temperature was maintained at $37^{\circ} \mathrm{C}$ by a heating pad.

\section{Evaluation of baroreflex responses}

Arterial pressure (AP) signals recorded from the femoral artery were processed by an arterial blood pressure analyzer (APR31a; Notocord, Croissy-Sur-Seine, France) and heart rate (HR) was derived instantaneously from the systolic blood pressure (SBP) signals. To evaluate brain stem cardiovascular regulation, the SBP signals were subject simultaneously to on-line and real-time spectral analysis (SPA10a; Notocord). We were particularly interested in the low-frequency (LF; $0.25-0.8 \mathrm{~Hz}$ ) component because it takes origin from RVLM [22], and its power density mirrors the prevalence of baroreflex-mediated sympathetic neurogenic vasomotor discharges that emanate from RVLM [23]. We also computed the baroreflex effectiveness index (BEI) based on online detection of spontaneous baroreflex sequences (BRS10a, Notocord), which were detected when SBP and pulse interval increased or decreased simultaneously [24]. As an index for baroreflex-mediated regulation of cardiac responses, BEI is defined as the total number of sequences divided by the total number of pressure ramps in each analysis zone.

\section{Intravenous administration of methamphetamine}

METH was obtained from the Food and Drug Administration, Department of Health, Executive Yuan, Taipei, Taiwan. METH and saline (vehicle control) were administered intravenously. Temporal changes in pulsatile AP, mean AP (MAP), HR, power density of the LF component of SBP signals and BEI were routinely followed for $240 \mathrm{~min}$ after intravenous administration of METH in an on-line and real-time manner [25,26]. The survival time and survival rate within 240 min were also recorded.

\section{Collection of tissue samples from brain and heart}

We routinely collected tissue samples from RVLM, CVLM, NTS, NACC, GPi, Cd, SN, VTA and heart at 20 or 240 min after the administration of METH or saline. Animals were killed with an overdose of pentobarbital sodium and neural tissues were collected bilaterally by micropunches according to their anatomical boundaries; cardiac tissues were collected directly from the left 
ventricle. The concentration of total proteins extracted from those tissue samples was determined by the BCA protein assay (Pierce, Rockford, IL, USA).

\section{Determination of METH in tissue samples}

The concentration of METH in cell lysate extracted from RVLM, CVLM, NTS, NACC, GPi, Cd, SN, VTA or heart was determined according to the manufacturer's protocol of a commercial METH indirect enzyme-linked immunosorbent assay (ELISA) kit (Calbiotech, Spring Valley, CA, USA), in conjunction with spectrophotometric determination (Multiskan Spectrum, Thermo Fisher Scientific Waltham, MA, USA) of the absorbance of reaction solution at $450 \mathrm{~nm}[27,28]$.

\section{Statistical analysis}

All values are expressed as mean \pm SEM. The temporal effects of METH or saline treatments on MAP, HR, power density of the LF component of SBP signals, BEI or tissue METH concentration were assessed using oneway or two-way analysis of variance (ANOVA) with repeated measures, as appropriate, for group means. In both cases, Scheffé multiple-range test was used for post hoc comparison of individual means. Mortality was assessed with the Fisher Exact Test. Correlation between tissue METH concentration in RVLM and BEI or power density of LF component of SBP signals was determined by linear regression analysis. $P<0.05$ was considered to be statistically significant.

\section{Results \\ Differential distribution of METH to brain and heart tissues after intravenous administration}

Figure 1 shows that on intravenous administration, METH (12 or $24 \mathrm{mg} / \mathrm{kg}$ ) exhibited a time-dependent distribution in brain and heart. While maintaining a dose-relationship, the concentration of METH in brain and heart tissues 20 min after administration of the two doses was approximately double that when measured at $240 \mathrm{~min}$. Detailed analysis revealed two interesting observations. First, the distribution to the heart, a commonly mentioned target for cardiovascular effects of METH $[1,29,30]$, was significantly less than brain tissues. Second, the concentration of METH was significantly higher in neural substrates associated with brain stem cardiovascular regulation (RVLM, CVLM or NTS) than brain targets for its psychological and neurological effects (NACC, GPi, Cd, SN or VTA).

\section{METH dose-dependently reduces survival}

Given at $12 \mathrm{mg} / \mathrm{kg}$, i.v. administration of METH elicited a $25 \%$ reduction in survival rate at $240 \mathrm{~min}$, which increased to $50 \%$ at a dose of $24 \mathrm{mg} / \mathrm{kg}$ (Figure 2).

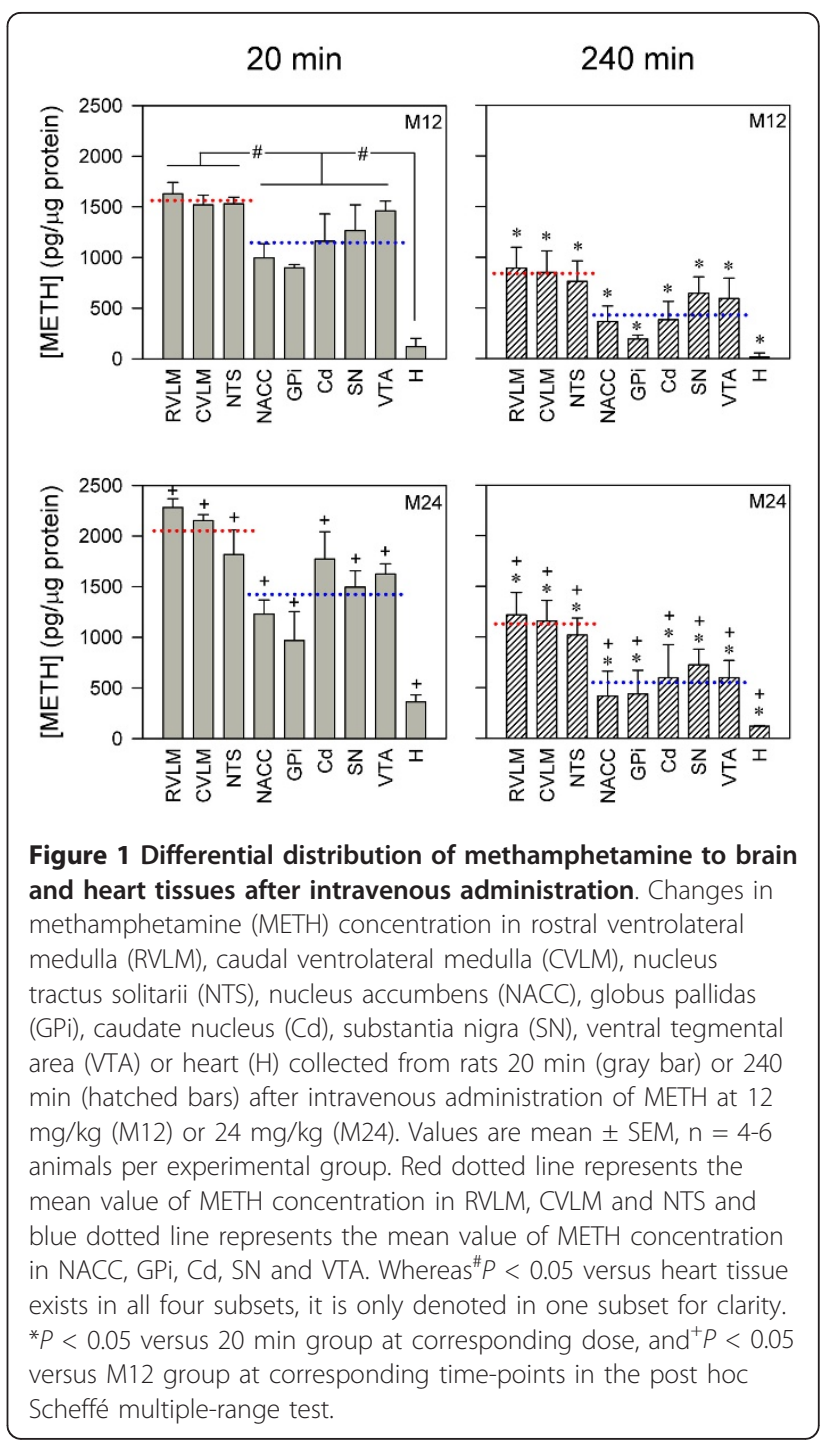

Similarly, METH (12 or $24 \mathrm{mg} / \mathrm{kg}$, i.v.) induced a dosedependent reduction in survival time (Figure 2).

\section{Depressed brain stem cardiovascular regulation in rats that survived METH intoxication}

The observation that METH manifested the highest concentration in RVLM, CVLM and NTS and the lowest concentration in heart among all tissues studied on systemic administration implies that this psychostimulant may effect circulatory depression not directly on the heart but indirectly via an action on the brain stem cardiovascular regulatory process. Our third series of experiments evaluated this possibility. In animals that survived METH (12 or $24 \mathrm{mg} / \mathrm{kg}$, i.v.), there was a significant and dose-dependent drop in MAP that peaked at $20 \mathrm{~min}$, followed by a gradual return to baseline (Figure 3$)$. HR was essentially maintained in rats that 

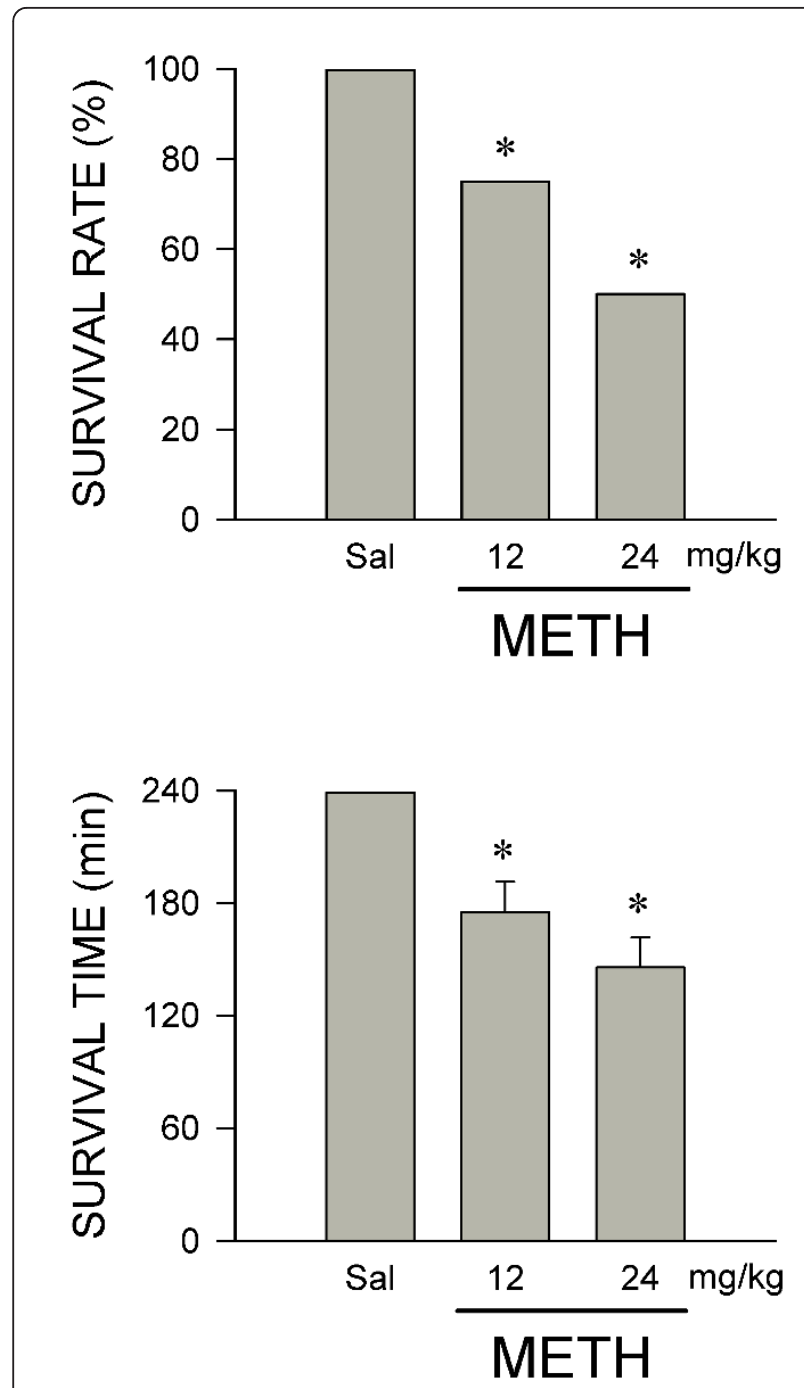

Figure 2 METH dose-dependently reduces survival rate and survival time. Dose-dependent changes in survival rate and survival time in rats that received intravenous administration of METH or saline (Sal, vehicle control). Values are mean \pm SEM, $n=5-16$ animals per experimental group at the beginning of the experiment. ${ }^{*} P<0.05$ versus saline group in the Fisher Exact Test.

received the lower dose of $\mathrm{METH}$, despite an initial drop at $20 \mathrm{~min}$. On the other hand, there was progressive bradycardia in animals that received the higher dose of METH, with a sustained HR at 200-240 min (Figure 3 ). Concurrent evaluation of BEI showed an initial augmentation that peaked at $40 \mathrm{~min}$ after the administration of METH (12 or $24 \mathrm{mg} / \mathrm{kg}$, i.v.). Whereas BEI returned to baseline in animals that received the lower dose, it underwent a graduate reduction that stabilized at 180-240 min after receiving the higher dose (Figure $3)$. On the other hand, with the exception of a transient increase at $20 \mathrm{~min}$ after administration of METH at 12

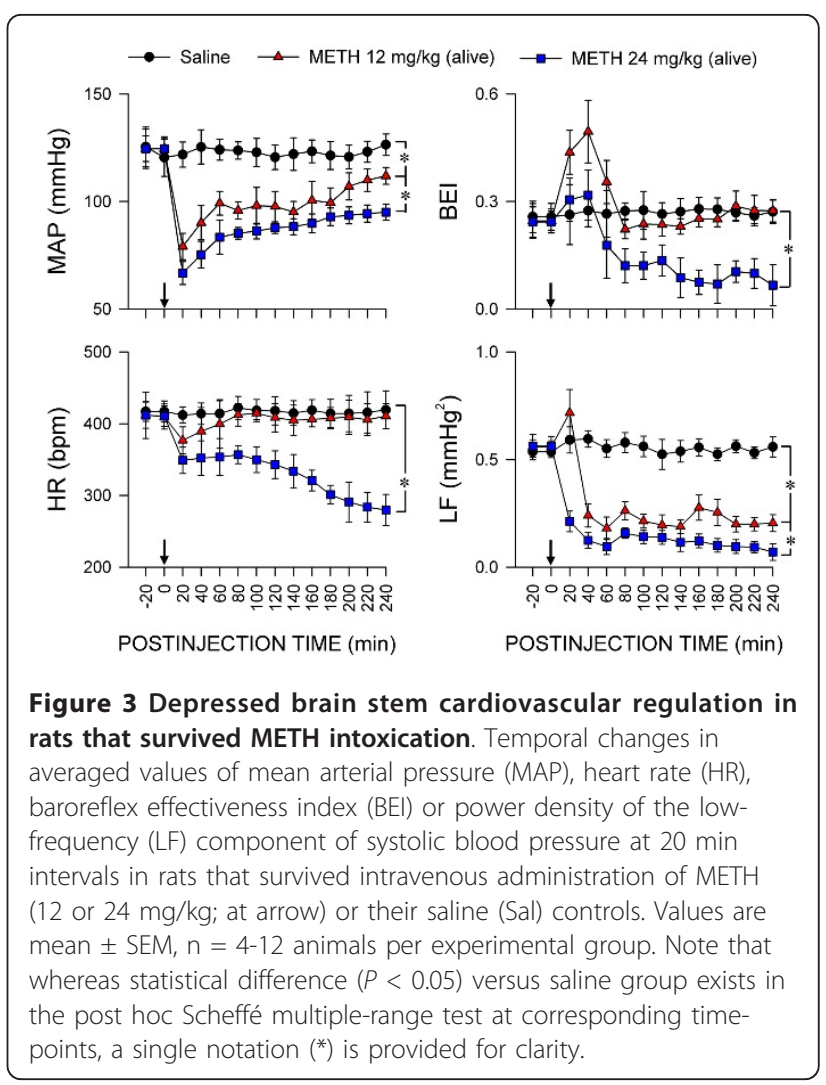

$\mathrm{mg} / \mathrm{kg}$, both doses elicited a progressive decrease in the power density of LF component of SBP signals over the first $60 \mathrm{~min}$. The LF power, however, lingered subsequently at a low level over the next $120 \mathrm{~min}$ (Figure 3).

Defunct brain stem cardiovascular regulation in rats that succumbed to METH intoxication

A drastically different picture was revealed in animals that succumbed to METH within 240 min after administration (Figure 4). The initial drop in MAP or HR during the first 20 min seen in animals that survived was followed by a continuous reduction that reached zero 180-220 min after METH administration (12 or $24 \mathrm{mg} / \mathrm{kg}$, i.v.). The initial augmentation of BEI gave way to immediate reduction that became zero at $180(24 \mathrm{mg} / \mathrm{kg})$ or $220(12 \mathrm{mg} / \mathrm{kg})$ min. Likewise, the LF power underwent an abrupt decrease and disappeared at 180-220 min. Of note is that the time course of disappearance of MAP, HR, BEI or power density of LF component of SBP signals (Figure 4) was commensurate with the survival time (Figure 2) induced by METH (12 or $24 \mathrm{mg} / \mathrm{kg}$, i.v.).

\section{Fatality from METH intoxication correlates with its distribution in brain}

Our final series of experiments addressed the issue of whether depressed or defunct brain stem cardiovascular 


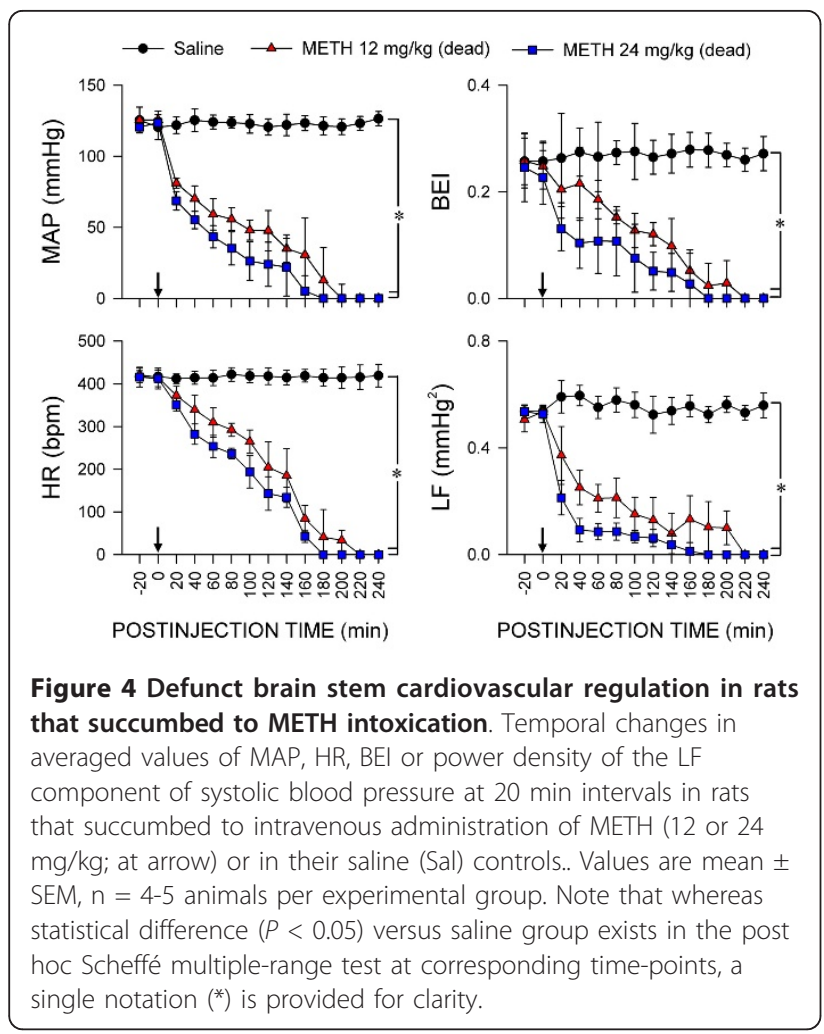

regulation, which dictates survival or death from METH, depends on the distribution of METH to brain stem nuclei that are associated with this homeostatic machinery. We found that the concentration of METH in RVLM, a key neural substrate in brain stem cardiovascular regulation, was positively and significantly correlated with the reduction in BEI and power density of LF component of SBP signals (Figure 5).

\section{Discussion}

The present study revealed two pieces of novel information regarding METH. First, on intravenous administration, this psychostimulant exhibited the largest distribution to brain stem nuclei that are associated with the baroreflex loop, followed by brain targets that are involved in the manifestation of its neurological and psychological responses; the concentration of METH in cardiac tissues was the lowest among all tissues studied. Second, METH effects acute cardiovascular depression by dampening the functional integrity of baroreflex, the degree of which correlated directly with its concentration in the relevant brain stem nuclei.

We chose to employ intravenous administration of METH in the present study because this is a common route [31] that is employed by abusers to rapidly raise the blood concentration of METH to euphoric levels. METH is more lipid-soluble than its primary metabolite

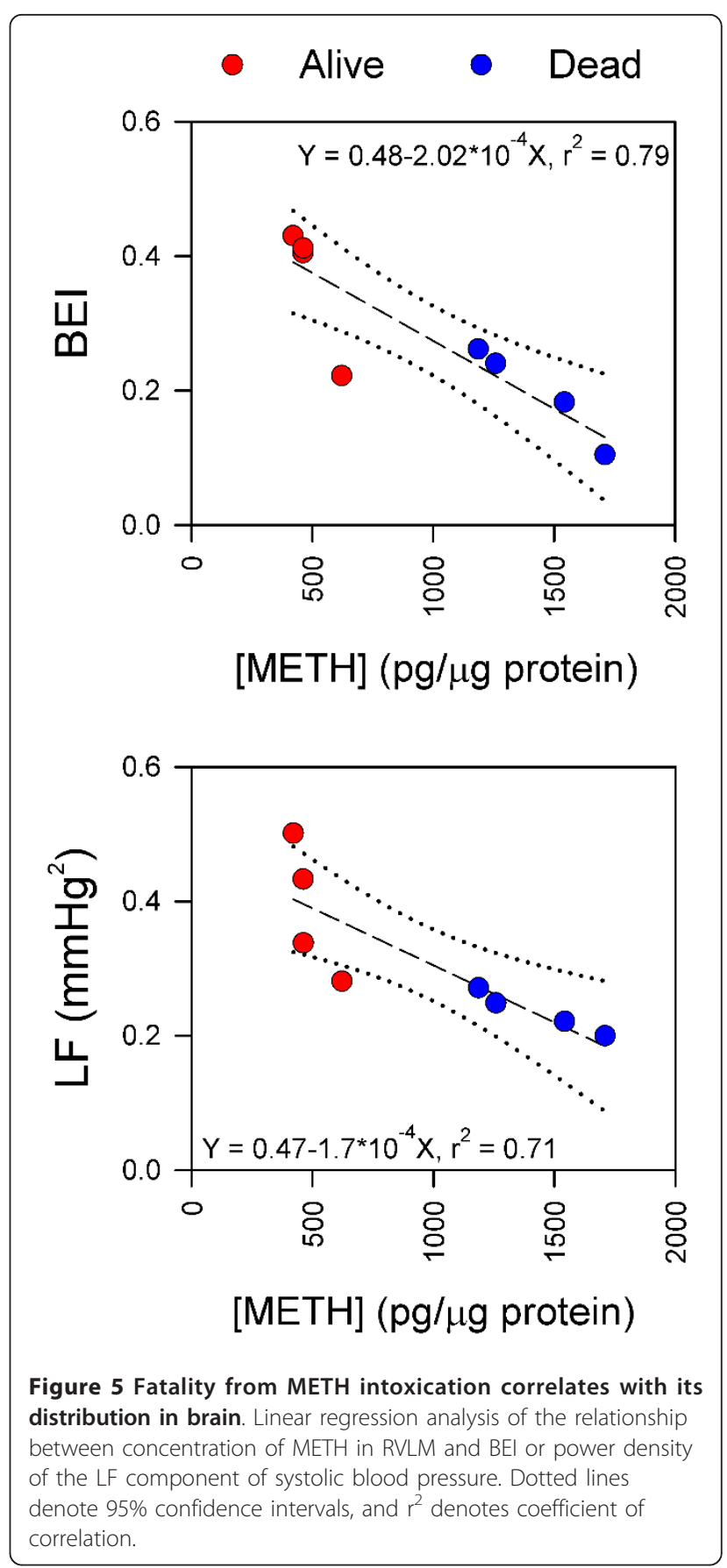

amphetamine, with enhanced transport across the blood-brain barrier and stability against enzymatic degradation by monoamine oxidase [32]. Whereas the dose-dependent and time-dependent pharmacokinetics data are in accordance with its chemical properties, we found that instead of NACC, GPi, Cd, SN and VTA, which are involved in the manifestation of its wellknown pharmacological effects, the highest concentration of METH was present in RVLM, CVLM and NTS, 
brain stem nuclei that are associated with the baroreflex circuitry. The ability to maintain a stable AP and HR in the form of baroreflex is essential to humans and animals alike. Inputs from primary baroreceptor afferents synapse at NTS. Outputs from NTS promote modulation of vagal and sympathetic outflow to the heart and peripheral vasculature via the CVLM and RVLM [20]. It is therefore intriguing that the preferential distribution of METH bears functional implications to METH intoxication. In animals that succumbed to METH, the baroreflex-mediated sympathetic vasomotor tone (LF power) and cardiac response (BEI) failed to function (defunct brain stem cardiovascular regulation), leading to the cessation of AP and HR. On the other hand, the power density of LF component of SBP signals and BEI, which either returned to baseline or decreased but stabilized above zero (depressed brain stem cardiovascular regulation) in animals that survived the two doses of METH studied, were sufficient to sustain AP and HR.

We recognize that METH may also act directly on the heart when administered intravenously, leading to heart failure in patients died of METH $[1,29,30]$. Whereas the design of our study does not allow us to repudiate this notion, the possibility for depression of cardiac functions, rather than failure of brain stem cardiovascular regulation, to be primarily responsible for METHinduced cardiovascular collapse is discernibly reduced by our observation that the distribution of METH to cardiac tissues was significantly less than to brain targets. Furthermore, successful resuscitation of an arrested heart depends on maintained functionality of brain stem cardiovascular regulation [19], suggesting that this homeostatic process, rather than the heart, holds the key to cardiovascular collapse.

METH abusers have to constantly increase dosing to sustain an elevated mood and libido and a decrease in appetite and fatigue. At the same time, continuous use at larger doses results in not only acute METH poisoning but also METH-induced sudden death $[16,29,30]$. Our novel pharmacokinetics findings offer a reasonable mechanistic underpinning for this phenomenon. We found that the extent that brain stem cardiovascular regulation is dampened is dependent on the concentration of METH in brain stem sites associated with this homeostatic process. An important ramification that arises from this observation is that accompanying the increase in dosing of METH and the sustained euphoric effects is the enhanced possibility for the depressed brain stem cardiovascular regulation to become defunct, leading to cardiovascular collapse. Since METH was determined in cell lysate extracted from the tissue samples, it is possible that our measurements simply represent accumulation of METH and bear no functional relevance. This possibility is deemed unlikely for at least two reasons. First, we demonstrated that the concentration of METH measured in RVLM, a key neural substrate in brain stem cardiovascular regulation, was positively and significantly correlated with the reduction in BEI and power density of LF component of SBP signals. Second, recent studies from our laboratory [33] indicated that the concentration of METH in the extracellular fluid collected by microdialysis from RVLM 10 min after intravenous administration is already 10 times higher than that in serum, approaching a plateau within $20 \mathrm{~min}$.

A majority of the literature on METH concerns primarily with behavioral alterations, physical dependence or psychopathology of withdrawal syndrome and its management that is associated with this psychostimulant. Much less studies are devoted to the mechanisms of cardiovascular collapse associated with METH intoxication despite that it is a common cause of death within the abuse population, $[14,15]$. The present study revealed that in addition to the brain targets that are associated with the reward system and neurodegenerative diseases, METH exhibits a preferential distribution to brain stem nuclei that are associated with cardiovascular regulation on intravenous administration. We further found that the concentration of METH in those brain stem sites dictates the extent that baroreflexmediated sympathetic vasomotor tone and cardiac responses are compromised, which in turn determines survival or fatality because of cardiovascular collapse. This information should offer novel leads for devising clinical management or developing therapeutic strategies against the increasing number of sudden death from METH abusers.

\section{Conclusion}

In conclusion, the present study revealed that by exhibiting the largest distribution to brain stem nuclei that are associated with the baroreflex loop on intravenous administration, METH effects acute cardiovascular depression by dampening the functional integrity of this fundamental homeostatic mechanism in brain stem cardiovascular regulation.

\section{Acknowledgements}

This work is supported by research grants from the Chang Gung Medical Foundation and the Chang Gung Memorial Hospital, Taiwan, Republic of China. [Grant number CMRPG891071 and CMRPG891072 to A.Y.W.C.].

\section{Author details}

${ }^{1}$ Center for Translational Research in Biomedical Sciences, Kaohsiung Chang Gung Memorial Hospital, Kaohsiung 833, Taiwan, Republic of China. ${ }^{2}$ Institute of Pharmacology, National Yang Ming University, Taipei 112, Taiwan,

Republic of China. 


\section{Authors' contributions}

FCHL performed the experiments. JCY participated in experimental design. SHHC and AYWC conceived the study, participated in experimental design, and drafted and revised the manuscript. All authors have read and approved the final manuscript

\section{Competing interests}

The authors declare that they have no competing interests.

Received: 10 November 2011 Accepted: 7 February 2012

Published: 7 February 2012

\section{References}

1. Chan P, Chen JH, Lee MH, Deng JF: Fatal and nonfatal methamphetamine intoxication in the intensive care unit. J Toxicol Clin Toxicol 1994 32:147-155.

2. Perez JA Jr, Arsura EL, Strategos S: Methamphetamine-related stroke: four cases. J Emerg Med 1999, 17:469-471.

3. Farrell M, Marsden J, Ali R, Ling W: Methamphetamine: drug use and psychoses becomes a major public health issue in the Asia Pacific region. Addiction 2002, 97:771-772.

4. Takayama N, lio R, Tanaka S, Chinaka S, Hayakawa K: Analysis of methamphetamine and its metabolites in hair. Biomed Chromatogr 2003, 17:74-82.

5. Chen CY, WU PN, Su LW, Chou YJ, Lin KM: Three-year mortality and predictors after release: a longitudinal study of the first-time drug offenders in Taiwan. Addiction 2010, 105:920-927.

6. Shaw KP: Human methamphetamine-related fatalities in Taiwan during 1991-1996. J Forensic Sci 1999, 44:27-31

7. Maxwell JC, Rutkowski BA: The prevalence of methamphetamine and amphetamine abuse in North America: a review of the indicators, 19922007. Drug Alcohol Rev 2008, 27:229-235.

8. Gehrke BJ, Harrod SB, Cass WA, Bardo MT: The effect of neurotoxic doses of methamphetamine on methamphetamine-conditioned place preference in rats. Psychopharmacology (Berl) 2003, 166:249-257.

9. Hatzipetros T, Raudensky JG, Soghomonian JJ, Yamamoto BK: Haloperidol treatment after high-dose methamphetamine administration is excitotoxic to GABA cells in the substantia nigra pars reticulata. Neurosci 2007, 27:5895-5902.

10. Dobbs LK, Mark GP: Comparison of systemic and local methamphetamine treatment on acetylcholine and dopamine levels in the ventral tegmental area in the mouse. Neuroscience 2008, 156:700-711.

11. Horner KA, Gilbert YE, Cline SD: Widespread increases in malondialdehyde immunoreactivity in dopamine-rich and dopamine-poor regions of rat brain following multiple, high doses of methamphetamine. Front Syst Neurosci 2011, 5:27-32.

12. Sonsalla PK, Albers DS, Zeevalk GD: Role of glutamate in neurodegeneration of dopamine neurons in several animal models of parkinsonism. Amino Acids 1998, 14:69-74.

13. Kaewsuk S, Sae-ung K, Phansuwan-Pujito P, Govitrapong P: Melatonin attenuates methamphetamine-induced reduction of tyrosine hydroxylase, synaptophysin and growth-associated protein-43 levels in the neonatal rat brain. Neurochem Int 2009, 55:397-405.

14. Shibata S, Mori K, Sekine I, Suyama H: Subarachnoid and intracerebral hemorrhage associated with necrotizing angiitis due to methamphetamine abuse-an autopsy case. Neurol Med Chir (Tokyo) 1991 31:49-52.

15. Kaye S, Darke S, Duflou J, McKetin R: Methamphetamine-related fatalities in Australia: demographics, circumstances, toxicology and major organ pathology. Addiction 2008, 103:1353-1360

16. Katsumata S, Sato K, Kashiwade H, Yamanami S, Zhou H, Yonemura I, Nakajima $\mathrm{H}$, Hasekura $\mathrm{H}$ : Sudden death due presumably to internal use of methamphetamine. Forensic Sci lnt 1993, 62:209-215.

17. Colfax G, Shoptaw S: The methamphetamine epidemic: implications for HIV prevention and treatment. Curr HIV/AIDS Rep 2005, 2:194-199.

18. Yu Q, Larson DF, Watson RR: Heart disease, methamphetamine and AIDS. Life Sci 2003, 73:129-140.

19. Chang AYW, Chan JYH, Chuang YC, Chan SHH: Brain stem death as the vital determinant for resumption of spontaneous circulation after cardiac arrest in rats. PLOS ONE 2009, 4:e7744.
20. Spyer KM: Central nervous mechanisms contributing to cardiovascular control. J Physiol 1994, 474:1-19.

21. Yang CH, Shyr MH, Kuo TBJ, Tan PPC, Chan SHH: Effects of propofol on nociceptive response and power spectra of electroencephalographic and systemic arterial pressure signals in the rat: correlation with plasma concentration. J Pharmacol Exp Ther 1996, 275:1568-1574.

22. Kuo TBJ, Yang CCH, Chan SHH: Selective activation of vasomotor component of SAP spectrum by nucleus reticularis ventrolateralis in rats. Am J Physiol 1997, 272:H485-H492.

23. Li PL, Chao YM, Chan SHH, Chan JYH: Potentiation of baroreceptor reflex response by heat shock protein 70 in nucleus tractus solitarii confers cardiovascular protection during heatstroke. Circulation 2001, 103:2114-2119

24. Laude D, Elghozi JL, Girard A, Bellard E, Bouhaddi M, Castiglioni P, Cerutti C, Cividjian A, Di Rienzo M, Fortrat JO, Janssen B, Karemaker JM, Lefthériotis $G$, Parati G, Persson PB, Porta A, Quintin L, Regnard J, Rüdiger H, Stauss HM: Comparison of various techniques used to estimate spontaneous baroreflex sensitivity (the EuroBaVar study). Am J Physiol Regul Integr Comp Physiol 2004, 286:R226-R231.

25. Chang AYW, Chan JYH, Cheng HL, Tsai CY, Chan SHH: Hypoxia-inducible factor $1 /$ heme oxygenase 1 cascade as upstream signals in the prolife role of heat shock protein 70 at rostral ventrolateral medulla during experimental brain stem death. Shock 2009, 32:651-658.

26. Chan SHH, Chan JYH, Hsu KS, Li FCH, Sun EYH, Chen WL, Chang AYW: Amelioration of central cardiovascular regulatory dysfunction by tropomyocin receptor kinase B in mevinphos intoxication model of brain stem death. Brit J Pharmacol 2011, 164:2015-2028.

27. Choi J, Choi MJ, Kim C, Cho YS, Chin J, Cho YA: The optimization of ELISA for methamphetamine determination: the effect of immunogen, tracer and antibody purification method on the sensitivity. Arch Pharm Res 1997, 20:46-52.

28. Kerrigan S, Phillips WH Jr: Comparison of ELISAs for opiates, methamphetamine, cocaine metabolite, benzodiazepines, phencyclidine, and cannabinoids in whole blood and urine. Clin Chem 2001, 47:540-547.

29. Saito T, Takeichi S, Nakajima Y, Yukawa N, Osawa M: Fatal methamphetamine poisoning in police custody. J Clin Forensic Med 1996, 3:183-185

30. Inoue H, Ikeda N, Kudo K, Ishida T, Terada M, Matoba R: Methamphetamine-related sudden death with a concentration which was of a 'toxic level'. Leg Med (Tokyo) 2006, 8:150-155.

31. Johnson BA, Roache JD, Ait-Daoud N, Wallace C, Wells L, Wang Y, Dawes MA: Effects of isradipine, a dihydropyridine-class calcium-channel antagonist, on d-methamphetamine's subjective and reinforcing effects. Int I Neuropsychopharmacol 2005, 8:203-213.

32. Cody JT: Determination of methamphetamine enantiomer ratios in urine by gas chromatography-mass spectrometry. J Chromatogr 1992, 580:77-95

33. Li FCH, Yen JC, Chan SHH, Chang AYW: Bioenergetics failure and oxidative stress in brain stem mediates cardiovascular collapse associated with fatal methamphetamine intoxication. PLOS ONE 2012, 7 : e30589.

doi:10.1186/1423-0127-19-16

Cite this article as: Li et al:: Defunct brain stem cardiovascular regulation underlies cardiovascular collapse associated with methamphetamine intoxication. Journal of Biomedical Science 2012 19:16. 